\title{
Correlated spin glass generated by structural disorder in the amorphous $\mathrm{Dy}_{6} \mathrm{Fe}_{74} \mathrm{~B}_{20}$ alloy
}

\author{
J. Tejada \\ Facultad de Física, Universitat de Barcelona, Diagonal 647, E-08028 Barcelona, Spain \\ B. Martinez \\ Instituto de Ciencia de Materiales de Barcelona-C.S.I.C., Martí i Franqués, \\ $s / n, 08028$ Barcelona, Spain \\ A. Labarta \\ Facultad de Física, Universitat de Barcelona, Diagonal 647, E-08028 Barcelona, Spain \\ E. M. Chudnovsky \\ Physics Department, Lehman College, The City University of New York, Bedford Park Boulevard West, Bronx, New York 10468
}

(Received 23 May 1991)

\begin{abstract}
Magnetic properties of the amorphous Dy-Fe-B alloy are studied in terms of the correlated-spin-glass approach of Chudnovsky et al. Features predicted by the theory are clearly observed in the experiment. It is shown that the magnetization law may be presented in the form where it is determined by the dimensionless correlation function of structural disorder, $C(y)$, only. The analysis of the magnetization curve allows one to distinguish between different models of disorder in amorphous solids. Experimental data on Dy-Fe-B are in favor of $C=\exp \left(-\frac{1}{2} y^{2}\right)$.
\end{abstract}

The structure of amorphous solids remains a challenging theoretical and experimental problem. While there are many models of amorphous disorder, ${ }^{1}$ one picture is commonly kept in mind. It displays the process of solidification as a diffusion-driven rearrangement of atoms towards the minimum energy state. The length of the structural order in a solid $R_{a}$ is, then, determined by the average size of the volume in which atoms can successfully rearrange until the diffusion coefficient becomes exponentially small as temperature goes down. Depending on the rate of cooling, one obtains solids ranging from monocrystals to disordered networks of atoms. Rapid solidification has been used to obtain a number of amorphous ferromagnetic alloys. In this paper we present a method that allows one to distinguish between different models of structural disorder in amorphous ferromagnets.

As was noticed long ago, the behavior of the magnetization on approach to saturation may be analyzed to elucidate structural properties of an inhomogeneous material. Becker and Doring, ${ }^{2}$ based upon early results for monocystals, ${ }^{3-5}$ showed that the magnetocrystalline anisotropy of randomly arranged crystallites gives a $\mathrm{H}^{-2}$ term in approaching saturation. Brown ${ }^{6}$ demonstrated that interaction of the magnetization with point, linear, and layered sources of spin pinning give $H^{-1 / 2}, H^{-1}$, and $H^{-2}$ contributions to the magnetization law, respectively. Chikazumi ${ }^{7}$ later noticed that the $H^{-1 / 2}$ law for localized pinning sources must change to $\mathrm{H}^{-2}$ in high fields. Vast literature on micromagnetism, which contains these and other results, has been reviewed by Brown $^{8}$ and Vonsovsky. ${ }^{9}$

In a more recent time, micromagnetic calculations were revived to describe amorphous ferromagnets. ${ }^{10-14}$ Instead of considering different types of spin pinning, a more universal approach has been applied, which operates with structural correlation functions. Magnetic properties of amorphous alloys are commonly described within the random anisotropy model. ${ }^{15,14}$ The basic idea of the model is that spin interactions in an amorphous ferromagnet are similar to these in a ferromagnetic crystal. The essential difference, however, is that instead of having global anisotropy axes, an amorphous ferromagnet has local easy axes whose direction $n(\mathbf{x})$ varies randomly in space. This direction is determined by the local arrangement of atoms. Consequently, directions of $n(\mathbf{x})$ at different points must rapidly become uncorrelated at distances greater than $R_{a}$. The corresponding correlation function $C(x)$ is of particular interest to us, since it reflects the short-range structure of amorphous solid. In the past magnetic measurements were used ${ }^{12,16}$ to obtain the value of $R_{a}$ in amorphous ferromagnets. The purpose of this work is to extract from magnetic measurements information about the form of $C(x)$.

Let $A(\mathrm{erg} / \mathrm{cm})$ and $K_{r}\left(\mathrm{erg} / \mathrm{cm}^{3}\right)$ be the exchange and random anisotropy strengths, respectively, averaged over the distance $R_{a}$. It has been shown ${ }^{13}$ that the magnetic behavior of the random anisotropy system changes drastically with the value of the dimensionless parameter $\lambda_{r}=(2 / 15)^{1 / 2}\left(H_{r} / H_{\mathrm{ex}}\right)$, where $H_{r}=2 K / M_{0}$ is the random anisotropy field, and $H_{\mathrm{ex}}=2 A / M_{0} R_{a}^{2}$. For $\lambda \gg 1$ (strong anisotropy) and low temperature, directions of spins are frozen along their local anisotropy axes. The arrow representation of this state is then similar to that of a spin glass. At $\lambda<1$ (weak anisotropy) the ferromagnetic correlation length $R_{f}$ becomes greater than $R_{a},{ }^{13}$ 
$R_{f}=R_{a} / \lambda^{2}$. In the absence of coherent anisotropy, $R_{f}$ remains finite at $T=0$ for however small $K_{r}$, in accordance with Imry-Ma arguments. ${ }^{17}$ This magnetic state has been called the correlated spin glass (CSG). ${ }^{13}$ Application of a magnetic field transforms it into a partially ordered state that has been called ferromagnet with wondering axes (FWA). ${ }^{18}$

In the case of a strong anisotropy, the approach to magnetic saturation follows a $1 / H^{2}$ law, independent of the form of $C(x) .{ }^{19}$ The case of the weak anisotropy is more subtle. ${ }^{11-13}$ Regardless of the form of $C(x)$, the magnetization law in the FWA follows $1 / \sqrt{H}$ at $H \ll H_{\mathrm{ex}}$ and $1 / H^{2}$ at $H \gg H_{\mathrm{ex}}$. In fact, the $1 / \sqrt{H}$ dependence, which is a characteristic of the FWA, has been observed in a number of systems. ${ }^{20-26,16}$ On the contrary, the $1 / H^{2}$ regime is more difficult to observe in weak anisotropy systems since it requires large magnetic fields. Information about $C(x)$ may be obtained from a measurement of $M(H)$ that includes $H \sim H_{\mathrm{ex}}$. In this paper we suggest such a study based upon the formula ${ }^{27}$

$$
\begin{aligned}
\frac{\delta M}{M_{0}}= & \frac{1}{30}\left[\frac{H_{r}}{H_{\mathrm{ex}}}\right]^{2}\left[\frac{H_{\mathrm{ex}}}{H}\right]^{1 / 2} \\
& \times \int_{0}^{\infty} d y C(y) y^{2} \exp \left[-y\left[\frac{H}{H_{\mathrm{ex}}}\right]^{1 / 2}\right],
\end{aligned}
$$

which is valid for the whole region approaching saturation; $y=x / R_{\alpha}$. Equation (1) allows one to obtain an explicit form of $C(y)$, performing an inversed Laplace transformation of the magnetization curve. This procedure, however, requires a greater accuracy in $M(H)$ measurement than has been achieved in our experiment. Instead, we have tried some typical forms of $C(x)$ in order to obtain the best fit of our experimental data.

Two important things should be mentioned before applying Eq. (1) to the study of $C(y)$. First concerns the inevitable presence of some coherent anisotropy in the sample. Although a weak coherent anisotropy, satisfying $\lambda_{c}^{1 / 2}>\lambda_{r}^{2} \quad$ [where $\lambda_{c}=(2 / 15)^{1 / 2}\left(H_{c} / H_{\mathrm{ex}}\right), H_{c}$ is the coherent anistropy field], destroys the CSG, ${ }^{13}$ the effect of $H_{c}$ becomes unimportant at $H>H_{c}$. Second, the field $H_{\text {ex }}$ must be accessible experimentally. For these reasons the amorphous Dy-Fe-B alloy has been selected, in which, according to our estimate (see below), $H_{\mathrm{ex}} \sim 1000$ Oe, $H_{r} \sim 1500 \mathrm{Oe}, \lambda_{r}^{2} \sim 0.3, \lambda_{c}^{1 / 2}<<0.3$.

A ribbon of $\mathrm{Dy}_{6} \mathrm{Fe}_{74} \mathrm{~B}_{20}$ was produced by the single roller technique in a closed chamber with a helium protective atmosphere. The melt was quenched on a $\mathrm{Cu}-\mathrm{Zr}$ wheel (diameter $200 \mathrm{~mm}$ ). The surface velocity was about $40 \mathrm{~m} / \mathrm{s}$. The amorphous state of the ribbon has been tested by x-ray and optical microscopy, and no evidence of crystalline inclusions has been detected. dc magnetic measurements have been carried out by using a commercial superconducting quantum interference device magnetometer with in-plane applied fields up to 50 $\mathrm{kOe}$ at $4.2 \mathrm{~K}$.

For the two field regimes discussed above, Eq. (1) gives

$$
\frac{\delta M}{M_{0}}=\frac{v_{c}}{30}\left(\frac{H_{r}}{H_{\mathrm{ex}}}\right)^{2}\left[\frac{H_{\mathrm{ex}}}{H}\right)^{1 / 2} \quad \text { if } H \ll H_{\mathrm{ex}},
$$

$$
\frac{\delta M}{M_{0}}=\frac{1}{15}\left[\frac{H_{r}}{H}\right]^{2} \text { if } H \gg H_{\mathrm{ex}},
$$

where

$$
v_{c}=\int_{0}^{\infty} d y y^{2} C(y)
$$

is a dimensionless number of the order of 1 . These two regimes have been observed experimentally (Fig. 1). One may notice a different slope at very high fields in Fig. 1(b). In that range of the field $\delta M$ becomes so small that the validity of Eq. (1) is violated by weak magnetic effects which have not been taken into account (the main effect, apparently, comes from the magnetism of conducting electrons). Equations (2) and (3), together with experimental curves, enabled us to estimate parameters $H_{r}$ and $H_{\text {ex }}$ (see above) and to verify self-consistency of the model.

The following procedure has been used to extract information about $C(y)$ from the magnetization curve. Let $S_{1 / 2}$ and $S_{2}$ be the slopes in Figs. 1(a) and 1(b) for $1 / \sqrt{H}$ and $1 / H^{2}$ regimes, respectively. With the help of Eqs. (2) and (3), Eq. (1) may be rewritten in the form
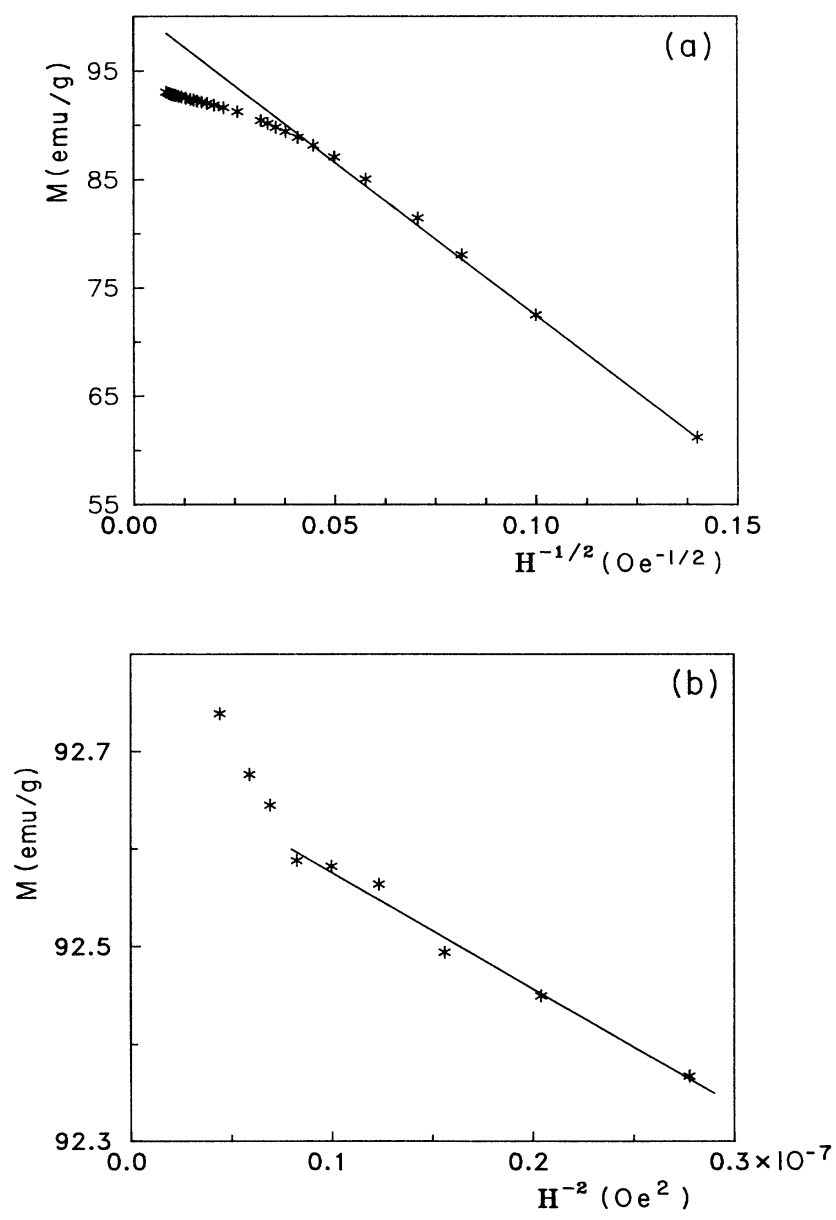

FIG. 1. Two regimes in approaching saturation: (a) $\delta M \propto 1 / \sqrt{H} ;$ (b) $\delta M \propto 1 / H^{2}$. 


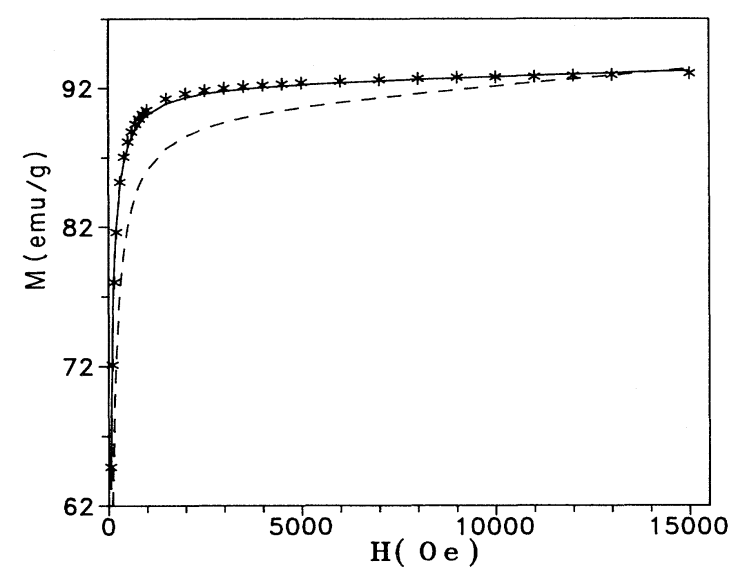

FIG. 2. Magnetization law in $\mathrm{Dy}_{6} \mathrm{Fe}_{74} \mathrm{~B}_{20}$ at $4.2 \mathrm{~K}$. The solid line corresponds to the fit by $C_{2}(y)=\exp \left(-\frac{1}{2} y^{2}\right)$. The dotted line shows the fit by $C_{1}(y)=\exp (-y)$.

$$
\begin{aligned}
M(H)=M_{0}-\frac{S_{1 / 2}}{v_{c} \sqrt{H}} \int_{0}^{\infty} & d y C(y) y^{2} \\
& \times \exp \left[-y\left(\frac{2 S_{1 / 2}}{v_{c} S_{2}}\right]^{1 / 3} \sqrt{H}\right] .
\end{aligned}
$$

A nice property of this formula should be noted. Since the parameters $M_{0}, S_{1 / 2}$, and $S_{2}$ are fixed by measurements at $H \ll H_{\mathrm{ex}}$ and $H \gg H_{\mathrm{ex}}, M(H)$ at $H \sim H_{\mathrm{ex}}$ depends only on the explicit form of $C(y)$, with no other fitting parameters. Note that this dependence is also hidden in the parameter $v_{c}$ Eq. (4). The function $C(y)$ satisfies $C(0)=1, C(y>1) \rightarrow 0 .{ }^{27}$ We have found that the fitting of the entire magnetization curve is quite sensitive to the rate of decay of the correlation function. One should also notice another important property of Eq. (5). It is invariant under a scale transformation, $y \rightarrow k y$. Consequently, the fit gives the functional form of $C(x)$; it is not sensitive to the value of $R_{a}$. As is shown in Fig. 2, a good fit is provided by the Gaussian form of the correlation function, $C(y)=C_{2}(y)=\exp \left(-\frac{1}{2} y^{2}\right)$. For comparison, we show the fit of $M(H)$ by $C_{1}(y)=\exp (-y)$ (dotted line), which is in apparent disagreement with experimental data.

For one who thinks about an amorphous state as formed of microcrystallites, $C(y)$ brings information about correlations in the orientation of locally defined crystallographic axes. $C_{2}(y)$ corresponds to a very fast decay of such correlations. Our observation that $C_{2}(y)$ satisfies the experimental data much better than $C_{1}(y)$ is, therefore, in favor of randomly oriented atomic clusters (microcrystallites) rather than a highly polygonized structure with a more gradual rotation of anisotropy axes. Notice, that for such a complex system as Dy-Fe-B, information about $C(y)$ can be hardly obtained by any other means, and is, thus, extremely helpful for any speculations on the nature of amorphous state in rapidly solidified alloys.

In conclusion, we have demonstrated that magnetic properties of amorphous Dy-Fe-B are well described by the random anisotropy model. An experimental method, based upon the approach of Chudnovsky and coworkers, ${ }^{13,14,18,27}$ is suggested, which allows one to distinguish between different models of structural disorder in amorphous ferromagnets. Application of this method to Dy-Fe-B indicates that its amorphous state is generated by the Gaussian decay of correlations in the orientation of atomic clusters.

We are grateful to Ron Dickman, Joel Lebowitz, and Jerome Percus for helpful discussions on amorphous disorder. E.C. acknowledges hospitality of the University of Barcelona and support from the NSF Grant No. DMR9024250 and PSC-CUNY Grant No. 661378.
${ }^{1}$ J. M. Ziman, Models of Disorder (Cambridge University Press, Cambridge, 1979).

${ }^{2}$ R. Becker and W. Doring, Ferromagnetismus (Springer, Berlin, 1939).

${ }^{3}$ P. Weiss, J. Phys. Rad. 9, 373 (1910).

${ }^{4}$ N. S. Akulov, Zs. Phys. 69, 794 (1931).

${ }^{5}$ R. Ganns, Ann. Phys. 15, 28 (1932).

${ }^{6}$ W. F. Brown, Jr., Phys. Rev. 58, 736 (1940).

${ }^{7}$ S. Chikazumi, Physics of Magnetism (Wiley, New York, 1964).

${ }^{8}$ W. F. Brown, Jr., Micromagnetics (Wiley, New York, 1963).

${ }^{9}$ S. V. Vonsovsky, Magnetism (Nauka, Moscow, 1980).

${ }^{10}$ R. Alben, J. J. Becker, and M. C. Chi, J. Appl. Phys. 49, 1653 (1978).

${ }^{11}$ M. Fahnle and H. Kronmuller, J. Magn. Magn. Mater. 8, 149 (1978).

${ }^{12}$ V. A. Ignatchenko, R. S. Iskhakov, and G. V. Popov, Zh. Eksp. Teor. Fiz. 82, 1518 (1982) [Sov. Phys. JETP 55, 878 (1982)].

${ }^{13}$ E. M. Chudnovsky and R. A. Serota, Phys. Rev. B 26, 2697 (1982); J. Phys. C 16, 4181 (1983).

${ }^{14}$ See also E. M. Chudnovsky, J. Appl. Phys. 64, 5770 (1988) for a review, and Refs. 16, 25, and 26 for the most recent experimental works.

${ }^{15}$ R. Harris, M. Plischke, and M. J. Zuckerman, Phys. Rev. Lett. 31, 160 (1973).

16J. Filippi, V. S. Amaral, and B. Barbara, Phys. Rev. B 44, 2842 (1991).

${ }^{17}$ Y. Imry and S. Ma, Phys. Rev. Lett. 35, 1399 (1975).

${ }^{18}$ E. M. Chudnovsky, W. M. Saslow, and R. A. Serota, Phys. Rev. 33, 251 (1986).

${ }^{19}$ E. Callen, Y. Liu, and J. R. Cullen, Phys. Rev. B 16, 263 (1977).

${ }^{20}$ D. J. Sellmyer and S. Nafis, J. Appl. Phys. 57, 3584 (1985).

${ }^{21}$ D. J. Sellmyer, S. Nafis, and M. J. O'Shea, J. Appl. Phys. 68, 3743 (1988).

22J. J. Rhyne, IEEE Trans. Magn. MAG-21, 1990 (1985).

${ }^{23}$ M. J. Park et al., Phys. Rev. B 33, 2070 (1986).

${ }^{24}$ W. A. Bryden et al., J. Appl. Phys. 61, 3661 (1987).

${ }^{25}$ P. M. Gehring et al., Phys. Rev. B 41, 9134 (1990).

26J. Tejada et al., Phys. Rev. B 41, 858 (1990).

${ }^{27}$ E. M. Chudnovsky, J. Magn. Magn. Mater. 79, 127 (1989). 